\title{
Pilot Study to Confirm that Fat and Liver can be Distinguished by Spectroscopic Tissue Response on a Medipix-All-Resolution System-CT (MARS-CT)
}

\author{
Kyra B. Berg ${ }^{1}$, James M. Carr ${ }^{2}$, Michael J. Clark², Nick J. Cook ${ }^{3}$, Nigel G. \\ Anderson $^{1}$, Nicola J. Scott ${ }^{4}$, Alexandra P. Butler ${ }^{1}$, Philip H. Butler ${ }^{2,5}$ \\ and Anthony P. Butler ${ }^{1,2,5,6}$
}

\begin{abstract}
${ }^{I}$ Department of Radiology, University of Otago, Christchurch, New Zealand, ${ }^{2}$ Physics and Astronomy, University of Canterbury, Christchurch, New Zealand, ${ }^{3}$ Department of Medical Physics, Canterbury District Health Board, Christchurch, New Zealand, ${ }^{4}$ Christchurch Cardioendocrine Research Group, University of Otago, Christchurch, New Zealand, ${ }^{5}$ European Organization for Nuclear Research, Geneva, Switzerland, ${ }^{6}$ Electrical and Computer Engineering, University of Canterbury, Christchurch, New Zealand
\end{abstract}

\begin{abstract}
NAFLD, liver component of the "metabolic" syndrome, has become the most common liver disease in western nations. Non-invasive imaging techniques exist, but have limitations, especially in detection and quantification of mild to moderate fatty liver. In this pilot study, we produced attenuation curves from biomedical-quality projection images of liver and fat using the MARS spectroscopic-CT scanner. Difficulties obtaining attenuation spectra after reconstruction demonstrated that standard reconstruction programs do not preserve spectral information.
\end{abstract}

Keywords: Medipix, spectroscopic x-ray imaging, computed tomography, fatty liver, non-alcoholic fatty liver disease. PACS: $87.57 . q h, 87.64 . \mathrm{kd}$

\section{INTRODUCTION}

We aim to demonstrate the potential of a novel spectroscopic-CT scanner to improve the evaluation of non-alcoholic fatty liver disease (NAFLD). NAFLD has become the most common form of liver disease in Western communities, affecting an estimated $17-33 \%$ of American adults (1). Primary NAFLD is the liver component of the "metabolic syndrome", a collection of diseases associated with obesity (2). The clinical spectrum of NAFLD ranges from simple fatty liver or steatosis, to non-alcoholic steatohepatitis (NASH, fatty liver with inflammation and evidence of hepatocyte damage), cirrhosis, and hepatocellular carcinoma or liver failure (1). In this pilot study, we demonstrate a method for measuring the attenuation curves of liver and fat tissue on a 3D X-ray imaging device with the anatomical resolution of a typical small animal microCT and with anticipated practical applications towards the quantification of fat in the liver.

NAFLD is traditionally diagnosed by invasive liver biopsy, with findings of macro-vesicular fat (2). Noninvasive techniques are available, all with limitations, including ultrasonography (US), computed tomography (CT), dual energy $\mathrm{CT}$, and magnetic resonance imaging (MRI) (3). Using US, only moderately severe fatty liver (greater than $30 \%$ ) can be detected, and fat content cannot be quantified (2). On $\mathrm{CT}$, fatty liver has a decreased attenuation compared to normal liver, and a threshold value of 10 HU below the spleen is used to qualitatively diagnose fatty liver. However, this criteria is not quantitative and is not sensitive to mild or moderate $(5-30 \%)$ fatty liver $(2,3)$. MRI is currently the most sensitive technique: fat can be detected and quantified using a T1-weighted gradient-echo in/out of phase sequence (3), but MRI is more expensive and less readily available than CT.

Dual energy CT has shown promise at beginning to address the sensitivity and quantification issues associated with single energy CT (4). A significant difference in attenuation between high and low X-ray tube voltage $(\Delta \mathrm{H})$ is seen for fatty liver but not for normal liver, and $\Delta \mathrm{H}$ is linearly correlated with percent fat (determined by histology) in rabbits (5) and humans (4). Dual-CT has been used to screen potential liver donors for fatty infiltration (6), however liver iron overload interferes with this measurement $(4,7)$. Since dual-CT has shown that energy information is 
valuable in diagnosing fatty liver, it is anticipated that spectroscopic-(multi-energy)-CT will improve our ability to quantify fat. The first step in investigating this would be to demonstrate the ability to extract the attenuation spectra of fat and liver from spectroscopic$\mathrm{CT}$ images.

X-ray attenuation curves have been measured for a variety of elements, compounds and mixtures, including adipose tissue, and are well understood (8). These curves are traditionally measured on a spectroscope, by scanning a monochromatic X-ray beam from a monochromator through a range of energies and measuring the absorption across that range. However, unless an expensive synchrotron Xray source is used, the beams are not intense enough to provide the spatial information required for biological imaging. The aim of this study is to produce attenuation curves from biomedical quality images of fat and liver tissues using the Medipix-2 spectroscopic detector in the MARS-CT scanner.

\section{METHODS}

\section{CT-Scan and Reconstruction}

Sheep liver and fat samples were prepared for scanning as follows: intact tissue was cut into cylindrical slices and packed tightly into a $25 \mathrm{cc}$ syringe. For the 50/50 liver/fat tissue blend, equal weights of each tissue were blended until homogenous, and the resulting paste was carefully piped into a $25 \mathrm{cc}$ syringe. All syringes of tissue were loaded into a perspex tube and scanned using the energy-sensitive photon counting pixel detector, Medipix-2 (pixel dimensions $55 \times 55 \mu^{2}$, detector size $14 \times 14$ $\mathrm{mm}^{2}$ ), taking 303 projections at 5 vertical detector positions and using each of the following 7 low energy thresholds: $12.5 \mathrm{keV}, 15 \mathrm{keV}, 17.5 \mathrm{keV}, 20 \mathrm{keV}, 25$ $\mathrm{keV}, 30 \mathrm{keV}, 35 \mathrm{keV}$. Acquisition times for each energy threshold were adjusted to give photon counts of approximately 1000 .

The reconstructions were performed with Octopus version 8.2. (9). Projection data was spot filtered (using the Octopus "normal (10)" setting) and transformed using cone beam reconstruction with linear interpolation and a regular Fourier filter. Reconstructions used a source detector distance (SDD) of $125 \mathrm{~mm}$, a source object distance (SOD) of $85 \mathrm{~mm}$, a centre of rotation of 759 pixels, and a vertical centre of 270 pixels.

\section{Constructing Attenuation Curves}

For ten projections of each sample at each of the seven low energy thresholds, the images were normalized by the acquisition time to obtain images in terms of photon counts per second. To obtain energy bins, the images for higher low energy thresholds were subtracted from the image for the low energy threshold directly below. On the subtracted images, a region of interest (ROI) corresponding to the tissue and to air (the open beam value) was selected and the average intensity taken. The tissue values were normalized by the air values and the negative logarithm was taken to get absorbance values. Finally, these values were divided by the average length of tissue the x-rays went through to get linear attenuation. Error was propagated from the standard deviation of the ROI using standard methods for all ten projections and combined to get an estimate of the natural variation of the tissue. The standard deviation of the calculated attenuation values across the ten projections (independent measurements) was taken as an estimate of the error in measurement.

\section{RESULTS AND DISCUSSION}

\section{Attenuation Curves from Spectroscopic- CT Projection Images}

To determine if attenuation spectra can be extracted from CT images, we performed a pilot study with sheep liver and fat. Liver, fat and a 50/50 weight to weight blend were scanned using seven different low energy thresholds. For ten projections of each sample, the images for different thresholds were subtracted to obtain images for energy bins. ROI's corresponding to the tissue and to air (the open beam value) were selected and average photon counts taken. From these values, linear attenuation was calculated. Here for the first time, we were able to construct linear attenuation curves directly from projection images taken on a CT scanner (Figure 1).

The data shows that the difference in linear attenuation between fat and liver is quite large, due to the difference in density of the tissues. However over this energy range the spectra themselves are not largely different by inspection. If we compare the ratio of attenuation values for fat and liver over the energy range we have measured, the attenuation of liver is always roughly twice that of fat. This is shown in Figure 1 by aiding comparison of the two curves using a constant scaling factor. This does not preclude significant differences between the spectra over the 10 $\mathrm{keV}$ to $100 \mathrm{keV}$ range. From the dual CT studies we know fat and liver differ at the higher energy end of 
the spectrum (5), and theoretically also at the lower energies where the k-edges of biological elements lie.

The estimated error in our measurement of linear attenuation (as measured over ten projection images, represented by the smaller error bars) does not impede distinction between liver and fat at any particular energy bin, and neither does the estimated variation in the actual tissue itself, (as measured by the variation in intensity over about 1000 pixels of the tissue, represented by the larger error bars). The difference in linear attenuation between fat and liver at low energy bins is quite large and it is linear attenuation that is represented on CT slices. In addition the liver/fat blend shows an intermediate difference in linear attenuation. While the curve for the $50 / 50$ blend is more similar to fat than liver, this is due to the greater volume of fat when a weight to weight $(\mathrm{w} / \mathrm{w})$ ratio is used. This study indicates quantitative information can be obtained by the use of narrow energy bins at low energies, as opposed to traditional broad spectrum CT. We have not addressed however in this pilot study the impact of variation from individual to individual, or variation over different types of fat deposits.

A Liver Fat Blend B
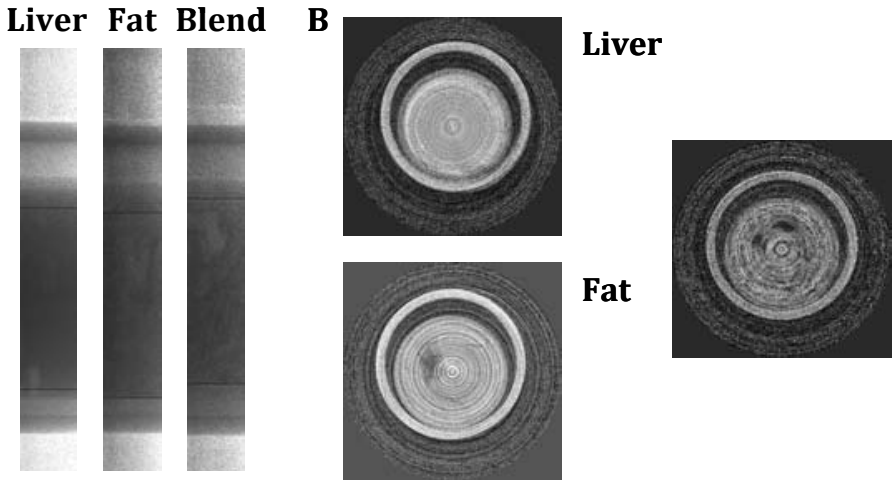

Blend

C

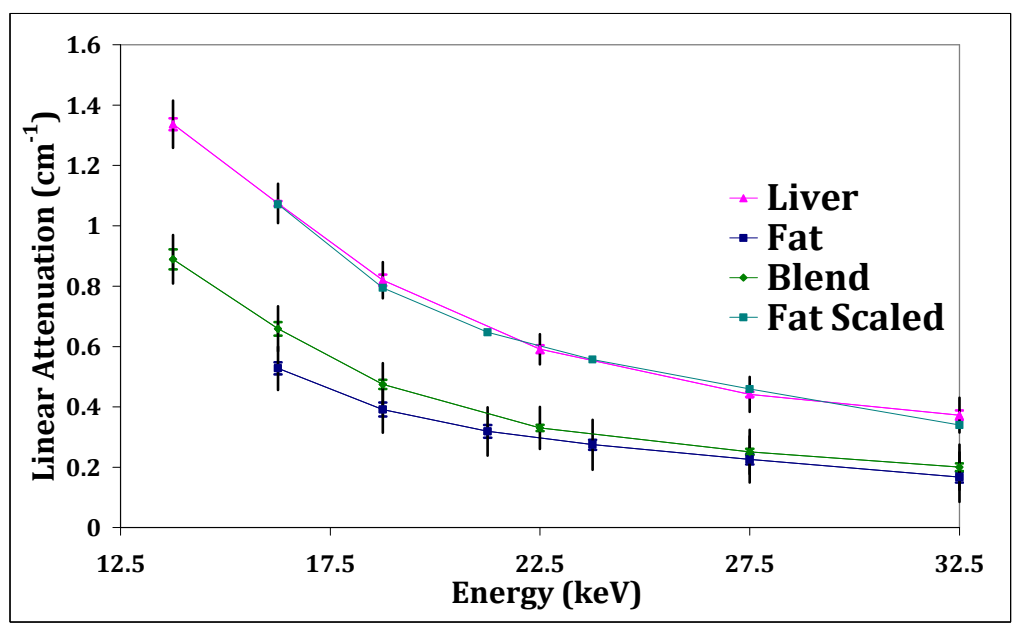

FIGURE 1. Construction of Liver, Fat and Liver/Fat Blend Linear Attenuation Curves from CT Images. A. Raw CT projection images of the three tissue preparations at $15 \mathrm{keV}$. B. Images reconstructed from 303 projections at $15 \mathrm{keV}$. C. Linear attenuation curves built from the projection images. Estimated error in measurement is shown by the smaller error bars, and estimated variation in the tissue is shown with the larger error bars.

To our knowledge, this is the first time attenuation curves have been constructed from biomedical-quality images. We have demonstrated that energy information, which can aid in distinguishing soft tissues such as liver and fat, can be extracted from projection images. Thus far, we have been unable to obtain accurate data from the reconstructed images with available cone beam reconstruction algorithms. There are likely several reasons for this. For one, the software used requires that images are converted in and out of various formats multiple times during the reconstruction and image processing. Preserving the 
spectral information contained in the images is difficult as the individual data sets are rescaled during these conversions. Simulations have indicated that multiple energy CT reconstructions can benefit from modified weightings during back projection (10) and other adaptations to traditional reconstruction techniques. Our future work in reconstruction will continue towards preserving spectral information for each and every voxel.

By demonstrating that we are able to measure fat and liver spectra with a small error in measurement, this pilot study establishes the potential to investigate differences in the spectra at alternative energy ranges with the MARS-CT scanner.

\section{Composition Analysis}

One proposed method of quantifying materials based on their spectral differences makes use of Principle Component Analysis (PCA) (11) and the Mahalanobis distance (12). For example, by conducting PCA on a sample of liver in multiple dimensions defined by attenuation over an energy range in which spectral differences from fat exist, we define the directions and magnitudes of maximum variance. This defines a region (in our multidimensional attenuation space) considered to be liver. Repeating this on a sample of fat, we can obtain a similar region considered to be fat. It is proposed that the Mahalanobis distance can then be used to quantify the likely ratio of fat and liver in any particular voxel. The magnitude of the Mahalanobis distance of the voxel data to either the liver or the fat regions gives a measure of likelihood that the voxel is fat and/or liver while the ratio of the Mahalanobis distances gives the ratio of fat to liver. There are also other methods currently being investigated for component analysis from spectral CT data. (13).

\section{CONCLUSIONS}

We have demonstrated the ability to simultaneously obtain spectral and biomedical-quality imaging information using the MARS-CT scanner. From projection images of liver, fat and a 50/50 blend, we were able to construct linear attenuation curves with a relatively small error in measurement.

Currently the spectral information is obtained from raw projection images, but by improving the software algorithms and data processing techniques the same information could be obtained from voxel data after reconstruction. This will allow spectral imaging of more complicated structures and for analysis of voxels based on spectral information by PCA. Further testing and exploration using the Mahalanobis distance and different energy ranges could lead to a method for quantifying fat/liver ratios based on their spectral differences. In addition it is worth investigating the use of the large difference in linear attenuation between liver and fat, enhanced by using low energy ranges and discrete energy bins.

\section{REFERENCES}

1. G. Farrell, C. Larter. Hepatology 43(2). S99-S112 (2006).

2. C. Lall, A. Aisen, N. Bansal, K. Sandrasegaran. AJR 190. 993-1002 (2008).

3. M. Karcaaltincaba, O. Akhan. Eur J Rad 61. 33-43 (2007).

4. V. Raptopoulos, A. Karellas, J. Bernstein, F. Reale, C. Constantinou, J. Zawacki. AJR 157. 721-5 (1991).

5. B. Wang, Z. Gao, Q. Zou, L. Li. Acta Rad 44. 92-97 (2002).

6. I. Kamel, J. Krustal, E. Pomfret, M. Keogan, G. Warmbrand, V. Raptopoulos. AJR 176. 193-200 (2000).

7. M.H. Mendler, P. Bouillet, A. Sidaner, E. Lavoine, F. Labrousse, D. Sautereau, B. Pillegand. J Hept 28. 785-94 (1998).

8. J.H. Hubbell, S.M. Seltzer. "Tables of X-Ray Mass Attenuation Coefficients and Mass-Energy Absorption Coefficients" in Physical Reference Data. NIST Standard Reference Database 126. $<$ http://physics.nist.gov/PhysRefData/XrayMassCoef/co ver.html >.

9. M. Dierick, B. Masschaele, L. Van Hoorebeke. Meas Sci Tech 15. 1365-70 (2004).

10. J. Giersch, D. Niederlöhner, G. Anton. Nucl Instr Met in Phys Res: Sec A 531(1-2). 68-74 (2004).

11. I.T. Jolliffe. Principal Component Analysis. Springer Series in Statistics $2^{\text {nd }}$ ed., New York: Springer, 2002.

12. P.C. Mahalanobis. "On the generalized distance in statistics" in Proceedings of the National Institute of Science of India, 1936, 2(1), pp. 49-55.

13. J.S. Butzer, A.P.H. Butler, P.H. Butler, P.J. Bones, N. Cook, L. Tlustos, "Medipix imaging: evaluation of datasets with PCA" in Image and Vision Computing New Zealand, $23^{\text {rd }}$ International Conference Proceedings, 2008, pp. 1-6. 